\title{
Haematopoietic Score for the Prognostic Evaluation of Multiple Myeloma Patients Undergoing First-line Treatment With a Bortezomib-based Regimen
}

\section{JingSong $\mathrm{He}$}

Zhejiang University School of Medicine First Affiliated Hospital

XiaoYan Yue

Zhejiang University School of Medicine First Affiliated Hospital XiaoYan Han

Zhejiang University School of Medicine First Affiliated Hospital DongHua He

Zhejiang University School of Medicine First Affiliated Hospital

Yi Zhao

Zhejiang University School of Medicine First Affiliated Hospital Yang Yang

Zhejiang University School of Medicine First Affiliated Hospital

\section{GaoFeng Zheng}

Zhejiang University School of Medicine First Affiliated Hospital

\section{WenJun Wu}

Zhejiang University School of Medicine First Affiliated Hospital

Zhen Cai ( $\nabla$ caiz@zju.edu.cn )

Zhejiang University School of Medicine First Affiliated Hospital https://orcid.org/0000-0001-6026-3804

\section{Primary research}

Keywords: Haematopoietic score, multiple myeloma, PFS, OS, prognosticpredictors

Posted Date: June 16th, 2020

DOl: https://doi.org/10.21203/rs.3.rs-35129/v1

License: (c) (i) This work is licensed under a Creative Commons Attribution 4.0 International License. Read Full License 


\section{Abstract}

Backgroud: It is very important to evaluate the prognosis of multiple myeloma (MM) patients before starting treatment. Although haematopoietic status may have a significant impact on patient survival, it has not received sufficient attention in current clinical practice.

Methods:This was a retrospective study of 150 newly diagnosed MM patients treated in one hematonosis treatment centre from May 2013 to June 2016 , all of whom received first-line treatment with a bortezomib-based regimen. The effects of haematopoietic factors, including haemoglobin levels (Hgb $<100 \mathrm{~g} / \mathrm{L})$, mean corpuscular volume $(\mathrm{MCV}>99.1 \mathrm{fL})$, and platelet count $(<150 \times 10 \mathrm{E} 9 / \mathrm{L})$, on the prognosis of the patients were analysed. Each of the above factors was assigned a value of 1 to generate a haematopoietic score.

Results: According to the results, 59 (39.3\%) patients had a score of 0, $43(28.7 \%)$ had a score of 1, 29 (19.3\%) had a score of 2, and 19 (12.7\%) had a score of 3. The median PFS times were 43.1, 24.5, 32.6 and 14.2 months, respectively $(P<0.001)$, and the median OS times were NR, 47.1 months, NR and 31.4 months, respectively $(\mathrm{P}<0.001)$. Multivariable analysis showed that the R-ISS stage (3 vs $1-2, \mathrm{HR}, 1.4)$, haematopoietic score $(3$ vs $0-2$, HR, 1.91$)$ and plasma cell percentage $(>30 \%, \mathrm{HR}, 1.96)$ are independent prognostic predictors for PFS; age ( $\leq 65$ years, HR, 0.54), the Durie-Salmon stage (3B vs 1-3a, $\mathrm{HR}, 2.96)$, haematopoietic score (3 vs $0-2, \mathrm{HR}, 2.53)$ and the bone marrow plasma cell percentage $(>30 \%, \mathrm{HR}, 2.35)$ are independent prognostic predictors of OS.

Conclusion: This study suggests that the haematopoietic score can be used to evaluate the prognosis of newly treated MM patients in the era of new drugs. However, there is still a need to enlarge the number of cases and carry out prospective research to validate this conclusion.

\section{Background}

Multiple myeloma(MM) is characterized by the clonal proliferation of malignant plasma cells, which can produce a large amount of monoclonal immunoglobulin (M protein). MM cells and their secreted M protein can cause a series of clinical symptoms, such as bone destruction, hypercalcaemia, anaemia, and renal insufficiency. A large number of new targeted drugs, such as proteasome inhibitors and immunomodulators, have entered clinical practice since 2000, significantly improving the efficacy of treatment for MM and increasing the median survival of patients from 3-5 years to 5-7 years(13).Currently, the proteasome inhibitor bortezomib and the immunoregulatory agents thalidomide and lenalidomide are the most important and commonly used drugs for the treatment of MM in China. Since February 2006, bortezomib has been used in our centres for the first-line treatment of Chinese MM patients ${ }^{(4-6)}$, with an overall response rate (ORR, partial remission and better) of $90 \%$; over $60 \%$ of patients achieve very good partial remission (VGPR) or better.

MM is an extremely heterogeneous disease, and survival can range from months to long-term stable disease. In general, it is very important to evaluate the prognosis of patients at the time of initial diagnosis. We previously utilized the Durie-Salmon staging system(6) and currently commonly use the International Staging System (ISS) and the Revised International Staging System (R-ISS); the latter has greater prognostic value in the new drug era(7, 8). In 2020, clinical data of MM patients treated at the Mayo Clinic in the United States for more than 10 years were retrospectively analysed(9), and the haematopoietic score, which considers the haemoglobin ( $\mathrm{Hgb}$ ) level, mean corpuscular volume (MCV) and platelet (PIt) count, on the patients' prognosis was found to have prognostic value. However, less than $45 \%$ of the patients in this group were treated with a proteasome inhibitor and/or immunomodulator-based regimen; therefore, the prognostic significance of the haematopoietic score needs further confirmation in the era of new drugs. The present study analysed the effects of the haematopoietic score and other factors on the prognosis of MM patients treated with bortezomib as firstline therapy, in the hope that this score will serve as an important supplement to the prognostic evaluation of newly diagnosed MM patients in the new drug era.

\section{Methods}

\section{Patients and treatment}

This study was a retrospective analysis and was approved by the Ethics Committee of the First Affiliated Hospital of the Medical School of Zhejiang University. From May 2013 to June 2016, patients who were newly diagnosed according to the World Health Organization (WHO) or International Myeloma Working Group (IMWG) diagnostic criteria in our centre were included if they had symptomatic MM with detectable M protein in their blood and/or urine and received first-line treatment with bortezomib-based regimen after being fully informed and providing signed informed consent. At least one course of treatment was completed, and the therapeutic efficacy was evaluated. From the beginning of treatment to the end of follow-up, all patients were informed of their condition and survival status through inpatient services, outpatient services or telephone contact.

All patients were evaluated based on the Durie-Salmon staging system and the ISS at the time of diagnosis.Using fluorescence in situ hybridization (FISH), bone marrow cells were examined for specific chromosomal abnormalities, including del-(17p13), 1q21 gain, del(13q14), and 14q32 rearrangement, and specific translocations, including $t(4 ; 14), t(11 ; 14)$, and $t(14 ; 16)$, were detected in a small number of patients with $14 q 32$ rearrangement. Retrospective assessment of the patients was based on the R-ISS, combining the patients' ISS stages, serum lactate dehydrogenase (LDH) levels, and FISH results, of which 1q amplification was also considered a genetic abnormality indicative of a poor prognosis(10).

All patients received a bortezomib-based regimen either as: a two-drug combined PD regimen (bortezomib combined with dexamethasone) or a threedrug combined regimen, with a third drug, such as cyclophosphamide (PCD), adriamycin (PAD) or thalidomide (PTD), added to the PD regimen. The 
specific administration method is detailed elsewhere ${ }^{(5)}$. If indicated by the patient's age, physical condition and willingness and if partial remission (PR) was obtained after at least 3-4 courses of induction treatment, autologous haematopoietic stem cell transplantation (ASCT) performed. After induction therapy with or without ASCT, patients received maintenance therapy based on bortezomib, lenalidomide, or thalidomide.

\section{Response}

The IMWG efficacy evaluation standard was used to assess the efficacy of treatment, including complete remission (CR), VGPR, PR, stable disease (SD) and progressive disease (PD)(11). Progression-free survival (PFS) was defined as the time from the beginning of the patients' first course of treatment to disease progression, death or the final follow-up visit. Overall survival (OS) was calculated from the time of the first course of treatment to death or the final follow-up visit.

\section{Data acquisition}

All patients were hospitalized during the initial diagnosis and treatment, and data regarding routine blood examinations, hepatic and renal function indicators, LDH levels, and bone marrow examination results were obtained from the hospital information system. The data obtained included the WBC count, Hgb level, MCV , platelet (PIt) count, serum creatinine level, serum LDH level, bone marrow plasma cell ratio and FISH examination results of bone marrow cells.

\section{Statistical analysis}

All patients were followed up until June 30, 2019, and the efficacy of treatment was evaluated after completing each course. The cut-off values for the Hgb level ( $<100 \mathrm{~g} / \mathrm{L})$; Plt count $(<150 \times 10 \mathrm{E} 9 / \mathrm{L})$ were based on the literature(2). The upper limit of normal for the MCV in our centre is $99.1 \mathrm{fL}$, which was selected as the threshold. The Kaplan-Meier method was used to generate survival curves, the log-rank test was used to compare differences in patient survival, and the chi-square test was employed for comparisons of classification data. Univariate analysis of age; Durie-Salmon stage; ISS stage, R-ISS stage; bone marrow plasma cell ratio; blood creatinine level; blood LDH level; peripheral blood WBC count, Hgb level, MCV, and Plt count; and the haematopoietic score generated using the last three variables were performed to examine the impacts of these variables on patient survival. All data test results were bilateral; P-values less than 0.05 were considered to be statistically significant, and factors with P-values less than 0.1 were then assessed by multivariate analysis. The results of multivariate analysis using the Cox proportional hazards model are shown as the hazard ratios (HRs) and $95 \%$ confidence intervals. The statistical analysis was performed using SPSS for Windows 20.0.

\section{Results}

\section{$\mathrm{Hgb}$ level, MCV and platelet count and patient characteristics}

A total of 150 newly diagnosed MM patients were enrolled in this study, of whom 76 were males and 74 were females; the median age was 62 years (range from 31 to 84 ). Table 1shows the baseline characteristics and treatment specifics of the patients.According to the results of the routine peripheral blood examinations at the time of diagnosis, 33 patients (22.0\%) had WBC counts lower than normal $(4 \times 10 \mathrm{E} 9 / \mathrm{L}), 101 \mathrm{patients}(67.3 \%)$ had an $\mathrm{Hgb}$ level lower than $100 \mathrm{~g} / \mathrm{L}, 37$ patients $(24.7 \%)$ had a MCV higher than normal $(99.1 \mathrm{fL})$ and 63 patients $(42.0 \%)$ had a Plt count lower than $150 \times 10 \mathrm{E} 9 / \mathrm{L}$.

A MCV higher than $99.1 \mathrm{fL}$ was common in patients with a $\mathrm{Hgb}$ level $<100 \mathrm{~g} / \mathrm{L}$ ( $32 \%$ vs $10 \%$ ) and bone marrow plasma cells percentage $>30 \%$ (39\% vs $12 \%$ ). A Hgb level $<100 \mathrm{~g} / \mathrm{L}$ was most common in patients aged $>65$ years ( $50 \%$ vs $20 \%$ ) and was also common inpatients with Plt counts $<150 \times 10 \mathrm{E} 9 / \mathrm{L}$ ( $84 \%$ vs $55 \%$ ); bone marrow plasma cells percentage >30\% (79\% vs $56 \%$ ); high-risk Durie-Salmon (76\% vs $72 \%$ vs $38 \%$ ), ISS ( $84 \%$ vs $75 \%$ vs $37 \%$ ) and RISS ( $20 \%$ vs $72 \%$ vs $81 \%$ ) stages; and elevated red blood cell volumes ( $86 \%$ vs $61 \%)$. A Plt count below $150 \times 10$ E9/L was common in patients with serum LDH levels above normal (63\% vs $37 \%$ ) and high-risk ISS stages (55\% vs $40 \%$ vs $28 \%$ ) (Supplementary Table 1 ).

\section{Haematopoietic score and patient survival}

The median duration of follow-up was 42.1 months, the median PFS time was 29.6 (21.8-37.4) months, and the estimated 3-year and 5-year PFS rates were $43.1 \%$ and $24.2 \%$, respectively. The median OS was not reached (NR), and the estimated 3-year and 5-year OS rates were $69.3 \%$ and $56.6 \%$, respectively. Patient age and bone marrow plasma cell percentage significantly affected patient PFS $(P<0.05)$, and Durie-Salmon stage, R-ISS stage and LDH levels also affected patient PFS ( $P<0.1)$ (Table 2,Supplementary Table 2). Moreover age, Durie-Salmon stage, ISS stage, R-ISS stage, bone marrow plasma cell percentage, and serum creatinine level had significant effects on OS $(P<0.05)$ (Table 3, Supplementary Table 2).

Based on univariable analysis, the Hgb level , MCV, and Plt count had significant effects on PFS and OS (Tables 2, 3, and Supplementary Table 2). The median PFS was 41.8 months for patients with Hgb levels $\geq 100 \mathrm{~g} / \mathrm{L}$ and 24.5 months for Hgb levels $<100 \mathrm{~g} / \mathrm{L}(P=0.063)$, and the median $0 S$ was NR and 64.0 months, respectively $(P=0.079)$. The median PFS was 32.6 months for patients with $\mathrm{MCVs}<99.1 \mathrm{fL}$ and 15.1 months for patients with MCVs $\geq 99.1$ $\mathrm{fL}(P=0.003)$, and the median OS times were NR and 33.2 months, respectively $(P<0.001)$. Additionally, the median PFS was 33.3 months for patients with Plt counts $\geq 150 \times 10 \mathrm{E} 9 / \mathrm{L}$ and 23.9 months for patients with Plt counts $<150 \times 10 \mathrm{E} 9 / \mathrm{L}(P=0.032)$, and the median OS times were NR vs 54.2 months, respectively $(P=0.022)$. Each of the above three indicators was assigned a score of 1 to generate the haematopoietic score. The integral values were 0,1 , 2, and 3, and the score significantly affected both PFS and OS ( $P<0.001)$ (Tables 2, 3 and Figure 1A, 1B). Overall, 59 (39.3\%), 43 (28.7\%), 29 (19.3\%) and 19 (12.7\%) patients had scores of 0, 1, 2 and 3, respectively; the median PFS times were 43.1 months, 24.5 months, 32.6 months and 14.2 months, respectively $(P<0.001)$, and the median OS times were NR, 47.1 months, NR and 31.4 months, respectively $(P<0.001)$. The median PFS was 32.6 months 
in patients who had a haematopoietic score from 0 to 2, and the estimated 3-year and 5-year PFS rates were $45.5 \%$ and $26.0 \%$, respectively. In patients with a score of 3, the median PFS was only 14.2 months for a score of 3, with estimated 3-year and 5-year PFS rates of $25.3 \%$ and $9.5 \%$, respectively $(P=$ 0.001) (Figure 2A, Table 1, Supplementary Table 2). The median OS was NR for patients who had a haematopoietic score from 0 to 2 , and the estimated 3-year and 5-year OS rates were $74.0 \%$ and $61.6 \%$, respectively. In contrast, the median OS of patients who had a score of 3 was only 31.4 months, with estimated 3-year and 5-year OS rates of 36.8\% and 23.7\%, respectively $(P<0.001)$ (Figure 2B, Table 2, Supplementary Table 2).

Multivariate analysis using the Cox proportional hazards model included age, Durie-Salmon stage, ISS stage and R-ISS stage, bone marrow plasma cell percentage, blood creatinine and LDH levels, and the haematopoietic score. The results suggested that R-ISS stage ( 3 vs $1-2, H R, 1.4 ; P=0.031)$, haematopoietic score ( 3 vs $0-2, H R, 1.91 ; P=0.033)$ and plasma cell percentage $(>30 \%, H R, 1.96 ; P=0.003)$ were independent prognostic predictors of $P F S$ and that patient age $(\leq 65$ years, $H R, 0.54 ; P=0.027)$, Durie-Salmon stage (3B vs $1-3 \mathrm{~A}, \mathrm{HR}, 2.96 ; \mathrm{P}=0.044)$, haematopoietic score $(3$ vs $0-2$, HR, 2.53; $\mathrm{P}=$ $0.006)$ and bone marrow plasma cells percentage $(>30 \%, \mathrm{HR}, 2.35 ; \mathrm{P}=0.005)$ were independent prognostic predictors of $\mathrm{OS}(\mathrm{Tables} 2,3)$.

\section{Discussion}

$\mathrm{MM}$ is a heterogeneous disease, and it is important to assess the prognosis of patients before the disease is treated. The Durie-Salmon staging system was once the most important staging system, and included the following factors: Hgb level, serum calcium level, extent of bone lesions, $\mathrm{M}$ protein level, and renal function status(6). However, this staging system is closely related to the tumour burden of MM, and its prognostic significance is often questioned in the era of new drugs. Currently, the ISS and the latest R-ISS are commonly applied in patientsundergoing first-line therapy. The former contains only two indicators, namely, the serum albumin and $\beta 2$-microglobulin levels, while the latter combines those with the serum LDH level and/or FISH-based cytogenetic abnormalities $(7,8)$. Anaemia is a common clinical symptom of MM and an indicator of a poor prognosis. In addition, abnormal red blood cell size, indicated by abnormal red blood cell distribution width (RDW) or average red blood cell volume values, and Plt counts are important MM prognostic indicators $(2,6,9,12-15)$, athough they have not received enough attention in current clinical practice.

Anaemia is very common in MM patients for a variety of reasons. For example, the infiltration of plasma cells into the bone marrow inhibits erythropoiesis, and the bone marrow microenvironment is altered; there is also a decline in the level of in erythropoietin due to renal insufficiency and anaemia owing to the release of various inflammatory factors that affect normal haematopoiesis. The role of Hgb in the prognosis of MM patients is clearly defined in the Durie-Salmon staging system, and $100 \mathrm{~g} / \mathrm{L}$ is used as one of the cut-off values for an abnormal Hgb level(6).

In addition, a previous Mayo clinic study (with patients enrolled from 1985 to 1988) found that a Hgb level $<100 \mathrm{~g} / \mathrm{L}$ and a Plt count $<150 \times 10 \mathrm{E} 9 / \mathrm{L}$ were predictive of relatively worse outcomes based on univariate analysis(2). However, those data were obtained for patients in the previous era of traditional chemotherapy. In this study, among patients treated with a bortezomib-based regimen, a Hgb level $<100 \mathrm{~g} / \mathrm{L}$ suggested worse PFS and OS, making it an important prognostic indicator for newly diagnosed MM patients.

Our data suggest that among patients treated with a bortezomib-based regimen, an increased MCV similarly suggests worse PFS and OS and that it occurs more often in patients with anemia, e.g., those with a $\mathrm{Hgb}$ level $<100 \mathrm{~g} / \mathrm{L}$ and bone marrow plasma cell percentage $>30 \%$. An increased red blood cell volume is often an indication of folic acid or vitamin B12 deficiency; however, it is regrettable that only a few patients in our group were tested for serum vitamin levels, with no deficiency found. According to the literature, vitamin B12 deficiency has been reported in approximately $13-20 \%$ of patients with plasma cell disease $(9,16,17)$. Regardless, this may not be related to macrocytosis in these patients, and some patients with vitamin B12 deficiency

do not show an increase in their $\operatorname{MCV}(9,16)$. It is believed that increased an erythrocyte volume is more common in elderly patients, which may be related to the shortened erythrocyte life span and the emergence of more new erythrocytes (reticulocytes) to replace the lost red blood cells, resulting in a slightly larger volume(18) and an increased peripheral blood red blood cell distribution width(12, 19).

The occurrence and development of MM depends on the tumour microenvironment in the bone marrow, and the interaction between myeloma cells and the bone marrow microenvironment is very complicated. The proliferation of MM cells, in addition to occupying the niches where haematopoietic cells can grow, results in the secretion of a large amount of haematopoietic inhibitory factors, which affect normal haematopoiesis(20, 21). Regarding megakaryocytic and erythroid precursor cells in particular, both the number and function are significantly inhibited, including clone formation ability and self-renewal ability(21). Therefore, low Hgb levels and Plt counts and an increased red blood cell volume may indicate the presence of more plasma cells in the bone marrow. Moreover, normal haematopoietic function is inhibited by various mechanisms, which indicates that the disease is more invasive. Our study showed that the peripheral blood Hgb level, MCV, and Plt count all affected PFS and OS in MM patients, while the WBC count did not. We also found that among patients older than 65 years old, with high-risk ISS and R-ISS stages, high bone marrow cell percentages and high serum LDH levels were more likely to occur when the Hgb levels were less than $100 \mathrm{~g} / \mathrm{L}$, the MCV was above the normal level and/or the PIt count was less than 150x10E9/L. The Hgb level, MCV, and Plt count are each assigned a score of 1 when they deviate from the given parameters, generating the haematopoietic score, which adequately predicts the survival of patients undergoing first-line treatment with a bortezomib-based regimen.

\section{Conclusion}

The haematopoietic score is simple and easy to obtain. Indeed, all three indicators can be obtained very readily from routine blood examinations. Furthermore, the score has significant prognostic value in patients receiving new drug treatments, indicating that it can be used as an important supplement to the R-ISS, especially when FISH cannot be routinely performed. However, this study was retrospective, and the number of cases was not large. Furthermore, blood vitamin B12 and folic acid levels were not detected. We intend to further expand the number of cases and re-evaluate the value 
of the haematopoietic score for prognostic prediction in MM patients in a more standardized prospective study and to combine cytogenetic abnormalities with the ISS; by doing so, we aim to establish a new prognostic system for MM patients that is applicable in the era of new drug therapy.

\section{Abbreviations}

MM, Multiple myeloma;ORR, objective remission rate; VGPR,very good partial remission; HR, hazard ratio;PFS, progression-free survival; D-S, DurieSalmon Staging; ISS, International Staging System; R-ISS, Revised International Staging System; Hgb, hemoglobin; PIt, platelet; WBC, white blood cell; MCV, mean corpuscular volume;WHO,the World Health Organization;IMWG, International Myeloma Working Group; FISH,fluorescence in situ hybridization; $\mathrm{LDH}$, lactate dehydrogenase; PD, bortezomib combine with dexamethasone; PCD, PD combine with cyclophosphamide; PAD, PD combine with adriamycin; PTD, PD combine with thalidomide; ASCT: Autologous haematopoietic stem cell transplantation;Cl, confidence interval; $\mathrm{Cr}$, creatinine; $\mathrm{NR}$, not reached.

\section{Declarations}

\section{Ethics approval and consent to participate}

Our study was approved by the Ethics Committee of the First Affiliated Hospital of the Medical School of Zhejiang University. Written informed consent of the patient was obtained.

\section{Consent for publication}

All authors agree with the final version of the manuscript and give their consent for its publication.

\section{Availability of data and materials}

The datasets used and/or analyzed during the current study are available from the corresponding author on reasonable request.

\section{Competing interests}

The authors declare no competing financial interests.

\section{Funding}

This study was supported by the Zhejiang Key Research and Development Project (grant number 2020C03014), and the National Major Scientific and Technological Special Project for "Significant New Drug Development" (grant number 2018ZX09733-003).

\section{Author contributions}

Jingsong He wrote part of manuscript, reviewed literature, manage the patient and collected clinical data; Xiaoyan Yuetranslated the manuscript, reviewed and wrote part of the manuscript;XiaoYan Han, DongHua He, Yi Zhao and GaoFeng Zheng reviewed and wrote part of the manuscript,suggested constructively; Yang Yang and WenJun Wu managed the patient, reviewed and wrote part of the manuscript; Zhen Cai wrote part of manuscript, review of literature andsuggested constructively. All authors reviewed the manuscript and contributed to the final draft.

\section{Acknowledgments}

None.

\section{Author details}

${ }^{1}$ Bone Marrow Transplantation Center, Department of Hematology, The First Affiliated Hospital, School of Medicine, Zhejiang University, Hangzhou, China

\section{References}

1. Palumbo A, Anderson K. Multiple Myeloma. N Engl J Med. 2011;364(11):1046-60.

2. Kyle RA, Gertz MA, Witzig TE, Lust JA, Lacy MQ, Dispenzieri A, et al. Review of 1027 patients with newly diagnosed multiple myeloma. Mayo Clin Proc. 2003;78(1):21-33.

3. Kumar SK, Rajkumar SV, Dispenzieri A, Lacy MQ, Hayman SR, Buadi FK, et al. Improved survival in multiple myeloma and the impact of novel therapies. Blood. 2008;111(5):2516-20.

4. Zheng W, Wei G, Ye X, He J, Li L, Wu W, et al. Bortezomib in combination with dexamethasone and subsequent thalidomide for newly-diagnosed multiple myeloma: a Chinese experience. Leuk Res. 2009;33(12):1615-8.

5. He J, Yang L, Han X, Zheng G, Zheng W, Wei G, et al. The choice of regimens based on bortezomib for patients with newly diagnosed multiple myeloma. PLoS One. 2014;9(6):e99174. 
6. B G Durie, 197 SES. A clinical staging system for multiple myeloma. Correlation of Measured Myeloma Cell Mass with Presenting Clinical Features, Response to Treatment, and Survival. Cancer. 1975;36(3):842-54.

7. Greipp PR, San Miguel J, Durie BG, Crowley JJ, Barlogie B, Blade J, et al. International staging system for multiple myeloma. J Clin Oncol. 2005;23(15):3412-20.

8. Palumbo A, Avet-Loiseau H, Oliva S, Lokhorst HM, Goldschmidt H, Rosinol L, et al. Revised International Staging System for Multiple Myeloma: A Report From International Myeloma Working Group. J Clin Oncol. 2015;33(26):2863-9.

9. Al Saleh AS, Sidiqi MH, Dispenzieri A, Kapoor P, Muchtar E, Buadi FK, et al. Hematopoietic score predicts outcomes in newly diagnosed multiple myeloma patients. Am J Hematol. 2020;95(1):4-9.

10. Rajkumar SV. Multiple myeloma: Every year a new standard? Hematol Oncol. 2019;37 Suppl 1(S1):62-5.

11. Durie BG, Harousseau JL, Miguel JS, Blade J, Barlogie B, Anderson K, et al. International uniform response criteria for multiple myeloma. Leukemia. 2006;20(9):1467-73.

12. Wang J, Xie X, Cheng F, Zhou X, Xia J, Qian X, et al. Evaluation of pretreatment red cell distribution width in patients with multiple myeloma. Cancer Biomark. 2017;20(3):267-72.

13. H; H, H; M, M; Y, F; M, T; S, A; N, et al. Red blood cell volume (MCV) as a new prognostic factor of multiple myeloma. Eur J Hoeinatol. 1995;54(1):57-8.

14. Kim DS, Yu ES, Kang KW, Lee SR, Park Y, Sung HJ, et al. Myeloma prognostic index at diagnosis might be a prognostic marker in patients newly diagnosed with multiple myeloma. Korean J Intern Med. 2017;32(4):711-21.

15. Kim SJ, Kim K, Kim BS, Jo DY, Kang HJ, Kim JS, et al. Clinical features and survival outcomes in patients with multiple myeloma: analysis of webbased data from the Korean Myeloma Registry. Acta Haematol. 2009;122(4):200-10.

16. Braschi C, Doucette J, Chari A. Characteristics of Vitamin B12 Deficiency in Patients With Plasma Cell Disorders. Clin Lymphoma Myeloma Leuk. 2017;17(12):e65-e9.

17. Baz R, Alemany C, Green R, Hussein MA. Prevalence of vitamin B12 deficiency in patients with plasma cell dyscrasias: a retrospective review. Cancer. 2004;101(4):790-5.

18. Gamaldo AA, Ferrucci L, Rifkind JM, Zonderman AB. Age-related changes in mean corpuscular volume in adult whites and African Americans. J Am Geriatr Soc. 2011;59(9):1763-4.

19. Liu S, Shi J, Guo H, Xu F, Wei M, Sun K, et al. Prognostic Significance Of The Inflammatory Index-Based Scoring System In Patients Preliminarily Diagnosed With Multiple Myeloma In The Bortezomib-Based Chemotherapy Era. Cancer Manag Res. 2019;11:9409-20.

20. Noll JE, Williams SA, Purton LE, Zannettino AC. Tug of war in the haematopoietic stem cell niche: do myeloma plasma cells compete for the HSC niche? Blood Cancer J. 2012;2(9):e91.

21. Bruns I, Cadeddu RP, Brueckmann I, Frobel J, Geyh S, Bust S, et al. Multiple myeloma-related deregulation of bone marrow-derived CD34(+) hematopoietic stem and progenitor cells. Blood. 2012;120(13):2620-30.

\section{Tables}


Table 1

Baseline characteristics of all patients

\begin{tabular}{ll}
\hline Baseline characteristics & $\mathrm{N}=150$ \\
\hline Age (years), median, (range) & $62(31-84)$ \\
Male, $\mathrm{n}(\%)$ & $76(50.7)$ \\
D-S, n (\%) & \\
\hline IA+2A+2B & $24(16.0)$ \\
\hline 3A & $97(64.7)$ \\
\hline 3B & $29(19.3)$
\end{tabular}

ISS, n (\%)

\begin{tabular}{ll}
1 & $43(28.7)$ \\
\hline 2 & $52(34.7)$ \\
\hline 3 & $55(36.7)$ \\
R-ISS, n (\%) & 122 \\
\hline 1 & $15(12.3)$ \\
\hline 2 & $75(61.5)$ \\
$\quad 3$ & $32(26.2)$ \\
Hgb (g/L), median, (range) & $89(44-157)$ \\
Plt, median, (range) & $158(36-513)$ \\
WBC, median, (range) & $5.1(1.9-14.1)$ \\
MCV (fL), median, (range) & $94.1(72.1-111.2)$
\end{tabular}

Hematopoietic score, n (\%)

\begin{tabular}{ll}
0 & $59(39.3)$ \\
\hline 1 & $43(28.7)$ \\
\hline 3 & $29(19.3)$ \\
\hline $\mathrm{DH}(\mu / \mathrm{L})$, median, (range) & $19(12.7)$ \\
\hline mol/L), median, (range) & $186.5(83-5785)$ \\
\hline
\end{tabular}

median, (range)

Therapy received, $\mathrm{n}$ (\%)

$\begin{array}{cc}\text { PAD } & 10(6.7) \\ \text { PCD } & 99(66.0) \\ \text { PTD } & 11(7.3) \\ \text { PD } & 30(20.0) \\ \text { ASCT, n (\%) } & 17(11.3)\end{array}$

Abbreviations: D-S, Durie-Salmon Staging; ISS, International Staging System; R-ISS, Revised International Staging System; Hgb, hemoglobin; PIt, platelet; WBC, white blood cell; MCV, mean corpuscular volume; LDH, lactate dehydrogenase; Cr, creatinine; PD, bortezomib combine with dexamethasone; PCD, PD combine with cyclophosphamide; PAD, PD combine with adriamycin; PTD, PD combine with thalidomide; ASCT: Autologous haematopoietic stem cell transplantation. 
Table 2

Univariable and multivariable analysis for PFS

\begin{tabular}{|c|c|c|c|c|c|}
\hline \multicolumn{3}{|l|}{ Univariable analysis } & \multicolumn{3}{|l|}{ Multivariable analysis } \\
\hline Variable & $\mathrm{HR}(95 \% \mathrm{Cl})$ & $P$ value & Variable & $\mathrm{HR}(95 \% \mathrm{Cl})$ & $P$ value \\
\hline Age $<65$ years & $0.72(0.48-1.06)$ & 0.096 & Age $<65$ years & $0.80(0.53-1.21)$ & 0.281 \\
\hline D-S & $1.53(0.94-2.49)$ & 0.082 & D-S & $2.19(0.88-5.43)$ & 0.091 \\
\hline \multicolumn{3}{|l|}{$3 B$ vs $1-3 A$} & \multicolumn{3}{|l|}{$3 B$ vs $1-3 A$} \\
\hline ISS & $1.26(0.84-1.90)$ & 0.265 & R-ISS & $1.40(1.03-1.89)$ & 0.031 \\
\hline \multicolumn{3}{|l|}{3 vs $1-2$} & \multicolumn{3}{|l|}{3 vs $1-2$} \\
\hline R-ISS & $1.52(0.93-2.48)$ & 0.092 & Hematopoietic score 3 vs $0-2$ & $1.91(1.05-3.46)$ & 0.033 \\
\hline \multicolumn{6}{|l|}{3 vs $1-2$} \\
\hline \multirow[t]{2}{*}{ WBC $<4 \times 10 E 9 / L$} & $1.33(0.84-2.11)$ & 0.220 & Plasma cells & $1.96(1.26-3.05)$ & 0.003 \\
\hline & & & \multicolumn{3}{|l|}{$>30 \%$} \\
\hline $\mathrm{Hgb}<100 \mathrm{~g} / \mathrm{L}$ & $1.50(0.98-2.30)$ & 0.063 & & & \\
\hline MCV > ULN & $1.89(1.23-2.91)$ & 0.003 & & & \\
\hline Plt & $1.54(1.04-2.28)$ & 0.032 & & & \\
\hline \multicolumn{6}{|l|}{$<150 \times 10 \mathrm{E} 9 / \mathrm{L}$} \\
\hline Hematopoietic score $0-2$ vs 3 & $2.38(1.39-4.09)$ & 0.001 & & & \\
\hline Plasma cells & $2.03(1.37-3.02)$ & $<0.001$ & & & \\
\hline \multicolumn{6}{|l|}{$>30 \%$} \\
\hline $\mathrm{Cr}>177 \mu \mathrm{mol} / \mathrm{L}$ & $1.37(0.79-2.37)$ & 0.264 & & & \\
\hline LDH $>$ ULN & 1.59 (0.99-2.53) & 0.051 & & & \\
\hline \multicolumn{6}{|c|}{$\begin{array}{l}\text { Abbreviations: HR, hazard ratio; Cl, confidence interval; D-S, Durie-Salmon Staging; ISS, International Staging System; R-ISS, Revised International } \\
\text { Staging System; WBC, white blood cell; Hgb, hemoglobin; MCV, mean corpuscular volume; ULN, Upper normal limit; PIt, platelet; Cr, creatinine; LDH, } \\
\text { lactate dehydrogenase. }\end{array}$} \\
\hline
\end{tabular}


Table 3

Univariable and multivariable analysis for OS

\begin{tabular}{|c|c|c|c|c|c|}
\hline \multicolumn{3}{|l|}{ Univariable analysis } & \multicolumn{3}{|l|}{ Multivariable analysis } \\
\hline Variable & $\mathrm{HR}(95 \% \mathrm{Cl})$ & $P$ value & Variable & $\mathrm{HR}(95 \% \mathrm{Cl})$ & $P$ value \\
\hline Age $<65$ years & $0.48(0.29-0.80)$ & 0.004 & Age $<65$ years & $0.54(0.31-0.93)$ & 0.027 \\
\hline D-S & $2.99(1.74-5.15)$ & $<0.001$ & D-S & $2.96(1.03-8.52)$ & 0.044 \\
\hline $3 B$ vs $1-3 A$ & & & $3 B$ vs $1-3 A$ & & \\
\hline ISS & $2.71(1.63-4.51)$ & $<0.001$ & R-ISS & $1.34(0.93-1.93)$ & 0.112 \\
\hline 3 vs $1-2$ & & & 3 vs $1-2$ & & \\
\hline R-ISS & $3.10(1.77-5.43)$ & $<0.001$ & Hematopoietic score 3 vs $0-2$ & $2.53(1.30-4.93)$ & 0.006 \\
\hline \multicolumn{6}{|l|}{3 vs $1-2$} \\
\hline \multirow[t]{2}{*}{ WBC $<4 \times 10 E 9 / L$} & $0.90(0.49-1.66)$ & 0.729 & Plasma cells & $2.35(1.29-4.27)$ & 0.005 \\
\hline & & & $>30 \%$ & & \\
\hline $\mathrm{Hgb}<100 \mathrm{~g} / \mathrm{L}$ & $1.70(0.95-3.05)$ & 0.071 & & & \\
\hline MCV >99.1fl & $2.53(1.51-4.26)$ & $<0.001$ & & & \\
\hline Plt & $1.75(1.06-2.90)$ & 0.027 & & & \\
\hline \multicolumn{6}{|l|}{ ه150x10E9/L } \\
\hline Hematopoietic score $0-2$ vs 3 & $2.98(1.64-5.43)$ & $<0.001$ & & & \\
\hline Plasma cells & $3.08(1.79-5.30)$ & $<0.001$ & & & \\
\hline \multicolumn{6}{|l|}{$>30 \%$} \\
\hline $\mathrm{Cr}$ & $2.81(1.56-5.06)$ & $<0.001$ & & & \\
\hline \multicolumn{6}{|l|}{$>177 \mu \mathrm{mol} / \mathrm{L}$} \\
\hline $\mathrm{LDH}>\mathrm{ULN}$ & $1.42(0.79-2.54)$ & 0.240 & & & \\
\hline $\begin{array}{l}\text { Abbreviations: PFS, progressio } \\
\text { R-ISS, Revised International St } \\
\text { lactate dehydrogenase; ULN, u }\end{array}$ & $\begin{array}{l}\text { rvival; HR, hazard } \\
\text { stem; WBC, white } \\
\text { nal limit. }\end{array}$ & $\begin{array}{l}\mathrm{Cl}, \text { confic } \\
\text { cell; Hgb, }\end{array}$ & $\begin{array}{l}\text { e interval; D-S, Durie-Salmon St } \\
\text { noglobin; MCV, mean corpuscul }\end{array}$ & $\begin{array}{l}\text { S, International St } \\
\text { e; PIt, platelet; } \mathrm{Cr} \text {, }\end{array}$ & $\begin{array}{l}\text { System; } \\
\text { hine; LDH }\end{array}$ \\
\hline
\end{tabular}

\section{Figures}

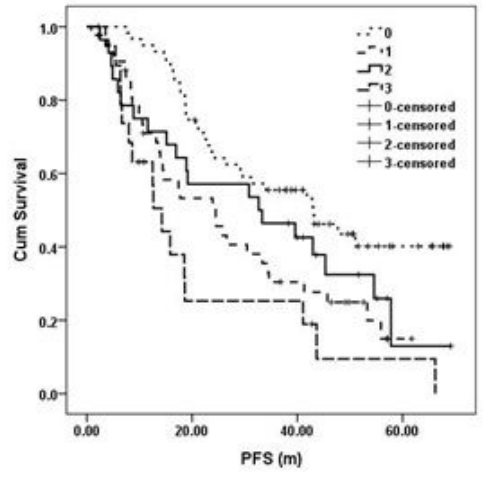

$1 \mathrm{~A}$

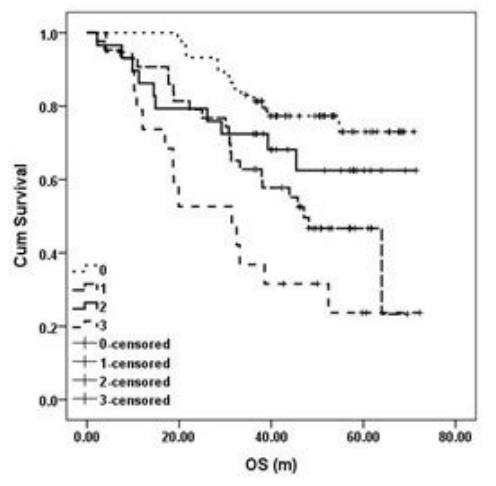

1B

\section{Figure 1}

Progression-free survival (1A) and overall survival (1B) based on the haematopoietic score $(0,1,2,3)$. PFS, progression-free survival; OS, overall survival. 


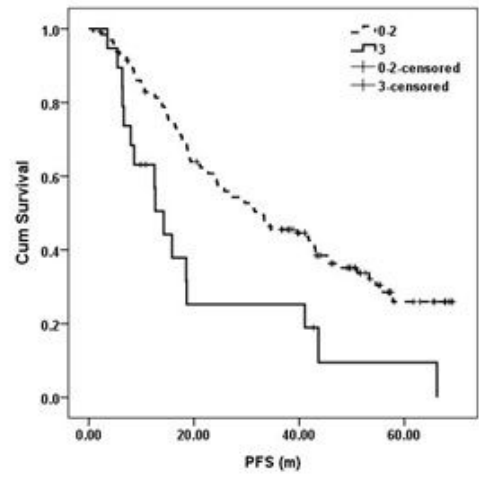

2A

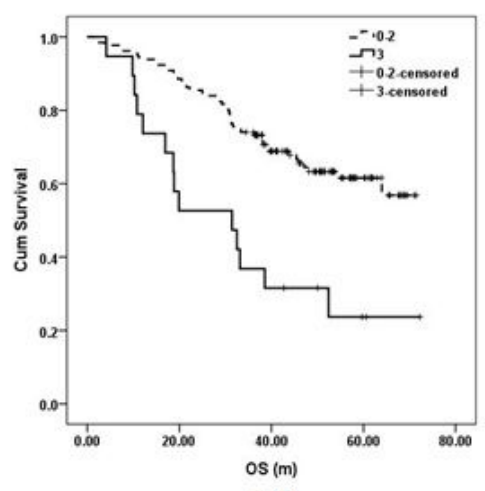

2B

Figure 2

Progression-free survival (2A) and overall survival (2B) based on the haematopoietic score $(0-2,3)$. PFS, progression-free survival; OS, overall survival

\section{Supplementary Files}

This is a list of supplementary files associated with this preprint. Click to download.

- SupplementaryTable.docx 